\title{
Proposta de Implementação de um Módulo de Re-roteamento Dinâmico de Túneis LSPs para a Ferramenta de Simulação GLASS
}

\author{
Klenilmar L. Dias, Klissiomara L. Dias, Kelvin L. Dias e Antônio Jorge G. Abelém
}

\begin{abstract}
Resumo- O GLASS (GMPLS Lightwave Agile Switching Simulator) é uma ferramenta de simulação para Redes Ópticas de Nova Geração, livre e de código aberto, desenvolvida em Java. Este artigo visa apresentar uma nova funcionalidade no simulador para facilitar o re-roteamento dinâmico de LSPs (Label Switched Paths) em cenários de redes GMPLS, baseado no protocolo de sinalização RSVP-TE.
\end{abstract}

Palavras chaves- Redes Ópticas, GLASS, MPLS, GMPLS, Simulação, Engenharia de Tráfego, Re-roteamento, LSP.

Abstract - The GLASS (GMPLS Lightwave Agile Switching Simulator) is a tool for simulationof next generation optical networks. It is based on free and open source code developed in JAVA. This paper aims at describing a new feature added to the GLASS, in order to facilitate the dynamic rerouting of LSPs (Label Switched Paths) in GMPLS networks scenarios based on the RSVP-TE signaling protocol.

Keywords - Optical Networking, GLASS, MPLS, GMPLS, Simulation, Traffic Engineering, Re-routing, LSP.

\section{INTRODUÇÃO}

A $\mathrm{s}$ tecnologías de redes têm evoluído muito rápido, tanto nos aspectos de capacidade de transmissão quanto no que diz respeito à confiabilidade, distâncias alcançadas, métodos de compartilhamento do meio e tipos de aplicações suportadas. Todo este desenvolvimento tem tido como principais forças motoras a evolução dos meios físicos, em particular, das fibras ópticas, o aprimoramento de técnicas de transmissão, a integração crescente de recursos multimídia às aplicações e o desenvolvimento da microeletrônica.

Em função da crescente convergência de aplicações e tipos de tráfego observados nas redes de comunicação, que têm capacidade limitada, os conceitos de qualidade de serviço e priorização de tráfego passaram a ser objetos de freqüente estudo. Hoje em dia, ainda não se dispõe de ferramentas completas para realizar os testes e análises para a

Klenilmar L. Dias, Programa de Pós-Graduação em Engenharia Elétrica, Universidade Federal do Pará, Belém, Brasil, E-mail: kldias@ seama.du.br.

Klissiomara L. Dias, Curso de Bacharelado em Sistemas de Informação, Faculdade Seama, Macapá, Brasil, E-mail: kdias@seama.edu.br.

Kelvin L. Dias, Faculdade de Engenharia de Computação, Universidade Federal do Pará, Belém. Brasil, E-mail: kld@ufpa.br.

Antônio Jorge G. Abelém, Faculdade de Informática, Universidade Federal do Pará, Belém, Brasil, E-mail: abelem@ufpa.br implementação de qualidade de serviço (QoS) em um sistema de comunicação.

Diante da carência de recursos para se criar laboratórios de sistemas de comunicação bem estruturados, diversos segmentos acadêmicos têm optado por testar e certificar propostas através de técnicas alternativas de avaliação de desempenho, em especial a simulação. $\mathrm{O}$ uso de simulação tem sido uma ferramenta poderosa no processo de projetar e avaliar arquiteturas, tecnologias e protocolos de comunicação de dados.

Outros meios de auxílio no processo de projetar e avaliar arquiteturas, tecnologias e protocolos de comunicação de dados consistem em avaliação analítica e experimentos práticos. A avaliação analítica é útil para demonstrar o impacto geral de uma determinada arquitetura em um cenário de comunicação de dados, os argumentos de entrada de um protocolo ou de mudança em condições específicas da rede. Porém, requer modelos simplificados da realidade, limitando o número de variáveis consideradas e as fontes de nãodeterminismo, já que os resultados são obtidos alterando argumentos em fórmulas.

Já os experimentos práticos, são baseados em um conjunto de execuções de um protocolo sobre um sistema de comunicação de dados real, portanto, produzem os resultados mais precisos. Apesar disso, devido à grande influência da topologia e dos sistemas e configurações envolvidos, os resultados coletados são dependentes de um cenário especifico, o que torna difícil sua reprodução em outro. Para protocolos, essa técnica requer que o mesmo já esteja implementado antes que seu funcionamento geral possa ser testado.

A simulação se encontra no nível intermediário, entre a avaliação analítica e os experimentos práticos. Ela permite que o projetista do cenário de comunicação de dados ajuste o nível de detalhe, atendo-se apenas aos recursos desejados [1].

No estudo das redes, especificamente das Redes Ópticas de Nova Geração, a simulação aparece muitas vezes como à única ferramenta que se pode usar com relativa facilidade, dada a dificuldade em reunir equipamentos suficientes para experimentar laboratorialmente topologias complexas e determinados cenários hipotéticos de tráfego. Contudo, mesmo em cenários e topologias mais simples o uso de técnicas de simulação é atrativo seja pela facilidade da coleta de dados e plotagem destes em forma de gráfico, bem como pela análise 
passo a passo dos diferentes protocolos de comunicação existentes. Através da simulação, podem ser feitos o projeto, dimensionamento e avaliação de desempenho de sistemas de comunicação de dados.

Existem diversos simuladores de tráfego de redes disponíveis, os que mais se destacam comercial e academicamente são o OPNET [2] e o Network Simulator (ns) [3]. O OPNET é um simulador comercial e proprietário largamente utilizado no âmbito corporativo, devido às suas funcionalidades e precisão nos resultados. Ele é mais utilizado em grandes empresas e operadoras de telecomunicações, mas restrito em outros ambientes, como o acadêmico, principalmente devido ao seu custo elevado.

$\mathrm{O} n s-2$ é utilizado principalmente por pesquisadores, por ter distribuição gratuita e código aberto. Tal fato o torna adequado a situações onde é necessário desenvolver novas funcionalidades, como em teses e projetos de pesquisa aplicada. No entanto, a sua interface (textual) não é amigável ao usuário. A execução de um experimento de simulação no $n s-2$ requer a elaboração de scripts em Tcl (Tool Control Language) e grande trabalho adicional para obter e visualizar os resultados. Também é comum que o usuário necessite programar em $\mathrm{C}++$ para que as funcionalidades desejadas estejam disponíveis. Além disso, os protocolos e tecnologias no $n s$-2, em geral, são desenvolvidos para uso isolado, para resolução de problemas específicos. Integrá-los, normalmente, é uma tarefa árdua.

As Redes Ópticas de Nova Geração são promissoras como mecanismo de suporte ao crescimento acelerado de tráfego. Essas redes, apesar de oferecerem uma grande quantidade de largura de banda, podendo levar a pensar que não há necessidade de gerenciá-las, apresentam problemas semelhantes quando comparadas com redes baseadas em pacotes.

Devido a esse fato, o MPLS (Multiprotocol Label Switching) [4] evoluiu para o GMPLS (Generalized MPLS) [5] para fornecer ao plano de controle (sinalização e roteamento) a possibilidade de encaminhar (comutar) em vários domínios diferentes, tais como domínios que utilizam pacotes (redes IP), tempo (TDM) e comprimento de onda (WDM). O GMPLS permite que a identificação de um determinado caminho (LSP - Label Switched Path) seja feita, por exemplo, através de comprimento de onda em uma rede óptica.

As redes ópticas estão em constante expansão para atender a demanda de informações requisitadas pelos novos aplicativos. Para evitar que essas expansões causem problemas ao seu funcionamento ou acabem sendo muito dispendiosas, é necessário estimar qual será o crescimento do tráfego na rede resultante. Obter uma estimativa confiável não é um procedimento trivial, mas é de fundamental importância para que o processo de expansão se torne eficiente.

Nesse contexto, um dos grandes problemas encontrados pelos alunos de graduação e pós-graduação nos cursos de Computação e Engenharia Elétrica é o alto custo de ferramentas de simulação para análise de protocolos e arquitetura de alto nível tolerante a falhas, em cenários de Redes Ópticas utilizando as tecnologias MPLS [4] e GMPLS [5].

O GLASS (GMPLS Lightwave Agile Switching Simulator) [6] é uma das poucas ferramentas voltada para simulação de Redes Ópticas de Nova Geração, livre e de código aberto e com uma interface amigável (gráfica) desenvolvida e implementada em JAVA, através de esforço conjunto dos grupos High Speed Network Technologies Group e Internetworking Technologies Group vinculados à Advance Network Technologies Division no NIST (National Institute of Standards and Technology) [7].

O GLASS tem uma estrutura de simulação, que permite a avaliação de protocolos de roteamento, restauração e sinalização em um ambiente de rede óptica e permite que os pesquisadores e projetistas de redes ópticas estudem o comportamento dos algoritmos e protocolos sem a necessidade de se construir um cenário real. Este simulador é a evolução do MERLin (Modeling Evaluation and Research of Lightwave Network) [8] uma ferramenta de simulação focada na avaliação e modelagem dos algoritmos de atribuição de rota e comprimento de onda em redes WDM.

O GLASS foi implementado utilizando como base a plataforma SSF (Scalable Simulation Framework) [9] que oferece componentes de redes como hosts, roteadores, enlaces de comunicação e alguns protocolos de redes.

O simulador utiliza a DML (Domain Modeling Language) [10] para projetar a topologia e gerar os scripts de simulação. A DML também permite um alto nível de descrição dos componentes e da configuração da topologia. Através desta linguagem é possível descrever os aspectos estáticos da rede, como a topologia da rede e suas propriedades, bem como os aspectos dinâmicos do cenário, que envolvem os protocolos que serão utilizados e os eventos que estão associados ao cenário.

Entretanto, um dos requisitos para se obter Engenharia de Tráfego em Redes Ópticas de Nova Geração com as tecnologias MPLS/GMPLS, é a capacidade de re-rotear LSPs (Label Switched Path) já estabelecidos na rede, sob certas condições, ou políticas administrativas. Outra característica importante é quando um LSP é re-roteado devido a uma falha em um circuito de comunicação, e quando essa falha estiver resolvida, o LSP deverá ser restabelecido pelo caminho original.

Atualmente, o GLASS na sua distribuição (versão 2.0.2) só tem incorporado o primeiro requisito descrito de Engenharia de Tráfego, isto é, re-rotear LSPs já estabelecidos na rede.

Este trabalho tem como objetivo apresentar a implementação do segundo requisito de engenharia de tráfego descrito, no simulador. O objetivo do novo módulo é fazer o restabelecimento dinâmico de LSPs primários, em caso da saída desses do estado de falha, visando o estudo da qualidade de serviço fim-a-fim para uma determinada aplicação em um cenário com as tecnologias MPLS/GMPLS.

O restante deste trabalho está organizado da seguinte forma. $\mathrm{Na}$ próxima seção são apresentados alguns trabalhos 
relacionados ao oferecimento de ferramentas de simulação. A Seção III descreve um resumo das características implementadas nos novos métodos inseridos no framework do simulador. Na Seção IV são apresentados o cenário e os experimentos de simulação para avaliar o novo módulo. Finalmente, a Seção V conclui o artigo e apresenta possíveis trabalhos futuros.

\section{TRABALHOS RELACIONADOS}

Existe uma quantidade considerável de trabalhos em simulação de redes de comunicação de dados, gerando várias ferramentas. Tais ferramentas podem ser classificadas em simuladores e ambientes de teste. Como exemplos de ambientes temos o Delayline [11], Dummynet [12], ComFIRM [13] e o x-sim [14]. Estas ferramentas não permitem que todas as condições sejam testadas, porque elas são limitadas por condições existentes.

Simuladores permitem que qualquer condição específica conhecida seja testada. Em geral, o projetista tem muito mais domínio sobre o experimento com simuladores do que com ambientes de teste. Porém, o modelo a ser simulado representa uma visão abstrata do protocolo real e da rede (como topologia, conectividade, perda de pacote, padrões de tráfego, etc.). Desenvolver um modelo de simulação envolve partir de premissas que simplifiquem o cenário de modo a focalizar-se nos aspectos relevantes do estudo. De qualquer forma, a precisão dos resultados da simulação depende de quão válidas são as premissas iniciais [15].

Como exemplos de ferramentas têm o trabalho apresentado em [16] que descreve o QNET, um simulador de redes IP desenvolvido com o objetivo de auxiliar trabalhos de pesquisa, projeto e gerenciamento de redes convergentes. O QNET fornece uma interface gráfica ao usuário, como o GLASS e o OPNET, para configurar os cenários e visualizar os resultados. Além disso, beneficia-se do potencial e flexibilidade proporcionados pelo $n s-2$. Entretanto, não possui implementação de módulo para redes ópticas MPL/GMPLS integrado à ferramenta, apesar do $n s-2$, possuir módulos de extensão para comutação por rótulos denominado MPLS Network Simulation (MNS) [17].

Em [18] é apresentado o Simmcast, um framework de simulação de eventos discretos baseado em processos que auxilia no projeto e avaliação de protocolos multicast. É baseado em Java e permite que os protocolos simulados sejam especificados utilizando um framework multi-thread orientado a objetos.

O trabalho apresentado em [19] descreve as arquiteturas física e funcional do protótipo RENATA (Redes Neurais Aplicadas ao Tráfego ATM). Esta ferramenta de simulação permite o desenvolvimento de agentes inteligentes, baseado em redes neurais, destinados à gerência pró-ativa de redes ATM (Asynchronous Transfer Mode).

Em [20], os autores descrevem uma nova versão para o trabalho apresentado em [19], chamado de RENATA 2, cuja evolução compreenderá o suporte à gerência pró-ativa de redes
IP, MPLS e GMPLS. Dentre possíveis tarefas realizadas pelos agentes gerados pelo ambiente RENATA 2 estão o controle de tráfego e alocação de caminhos entre os hosts de um sistema de comunicação. Porém, não implementa o re-roteamento dinâmico de túneis MPLS.

Em [21] é apresentada uma análise comparativa de algumas das principais ferramentas de simulação utilizadas para avaliação de desempenho de redes e projetos de protocolos. Neste trabalho foram selecionadas, para estudos mais aprofundados, três destas plataformas de simulação: NS-2, GloMoSim e NCTUns.

Com o estudo dos trabalhos citados acima e de outros encontrados na literatura, observa-se pouca preocupação no desenvolvimento ou aprimoramento de ferramentas de simulação que permita modelar e avaliar o desempenho de protocolos de roteamento, restauração e sinalização tolerantes a falhas em redes ópticas com as tecnologias MPLS/GMPLS.

Este artigo contribui com o acoplamento de um novo módulo com característica de engenharia de tráfego e baseado nas principais classes das mensagens PATH e RESV do protocolo de sinalização RSVP-TE (Resource Reservation Protocol - Traffic Engineering) para fazer o re-roteamento dinâmico de túneis LSPs.

\section{DESCRIÇÃO DA IMPLEMENTAÇÃO}

Por questões de legibilidade, o diagrama de classes apresentado na Figura 1 ilustra apenas as principais classes e alguns métodos diretamente envolvidos na solução. Para ser possível a inserção do novo módulo de re-roteamento, inicialmente foi necessário realizar ajustes no código (na versão distribuída do simulador) para que este compilasse perfeitamente, por exemplo, chamadas de métodos que não existiam ou existiam com outro nome. A técnica de Engenharia Reversa se fez necessária para um melhor entendimento dos pacotes implementados, a partir do qual foi possível evoluir o simulador, inserindo a funcionalidade apresentada neste artigo.



Fig. 1. Principais clases e métodos participantes da solução

\section{A. Classe TEAgent}

O TEAgent é a classe de Engenharia de Tráfego do simulador que faz a verificação dos eventos das mensagens 
PATH Message e RESV Message do protocolo RSVP-TE. Esta classe possui um método chamado callback que fica de tempos em tempos verificando eventos de falhas ou recuperação no circuito de comunicação MPLS/GMPLS. O método callback recebe como parâmetros as mensagens oriundas dos eventos que podem ocorrer. Na ocorrência de qualquer um dos casos, é feito o reporte do evento ao protocolo de sinalização RSVPTE, passando como parâmetro informações da interface do elemento que deve ser recuperado. Como parte deste processamento, é feita uma chamada ao método recoverReportFromAgent da classe $R S V P$, o qual é explicado a seguir.

\section{B. Classe RSVP}

As mensagens de PATH e RESV do Protocolo RSVP-TE são trocadas entre os elementos de rede para estabelecerem um caminho, ou LSP. Os Objetos utilizados pelas mensagens de $R E S V$ e $P A T H$ estão inseridos na classe $R S V P$.

\section{1)Método recoverReportFromAgent}

O método recoverReportFromAgent é um método abstrato ${ }^{1}$ herdado da classe SignalingProtocol. Por este motivo, o mesmo teve de ser sobrescrito na subclasse $R S V P$, a fim de implementar o processamento desejado. Desta forma, o processamento implementado tem como função principal executar a recuperação do túnel LSP. A seguir são descritas as principais características do novo código implementado nesse método.

a) Obtenção de um vetor contendo informações acerca dos LSPs que devem ser recuperados.

b) Obtenção das informações necessárias à mensagem RESV. Enviado em resposta às mensagens $P A T H$, mas usado também para remover reservas da rede.

c) Construção de objeto contendo o endereço IP do elemento (ex: OXC -Optical Cross Connect) que vai ser recuperado, o código indicando que se trata de recuperação, o tempo de inicio da recuperação, o ID do nó a ser recuperado, entre outras informações necessárias.

d) Verificação se o LSP, no momento, é de ingresso ou egresso. Se for ingresso, é dado início ao processo de reroteamento. Se egresso, gera uma notificação MPLS informando como parâmetros: objetos da classe SessionObject, o objeto montado no item c, um valor booleano (true se upstream e false se downstream) e um objeto do tipo ClassObect. Para tanto, invoca o método genNotificationMsg da classe Message. Se o LSP for de ingresso é realizada a verificação do tipo de componente, no caso OXC, e em seguida o método OXCTypeRecover da classe TEAgent é invocado.

O método OXCTypeRecover delega parte do seu processamento para um novo método criado na classe MPLSForwardingTable

chamado

\footnotetext{
${ }^{1}$ Método declarado apenas com a assinatura (tipo de retorno, nome e lista de parâmetros), sem processamento algum, em uma classe abstrata. Subclasses que herdam da classe que declara um método abstrato devem sobrescrever (overriding) o método abstrato, caso desejem fornecer processamento específico.
}

reRouteToPrimaryPathLSP_OXC. Este método é explicado na Seção $C$.

\section{2)Método sendPrimaryPathOnToEgress}

$\mathrm{O}$ método sendPrimaryPathOnToEgress foi criado com o objetivo de auxiliar o método reRouteToPrimaryPathLSP_OXC, localizado na classe MPLSForwardingTable, no re-roteamento de túneis LSPs primário. $\mathrm{O}$ método sendPrimaryPathOnToEgress recebe como parâmetro o LSP primário e seu respectivo backup. Dessa forma, ao encontrar o LSR de egresso envia uma mensagem de notificação PATH Message e não mais RESV Message. Em seguida, invoca o método rcvPrimaryPathOnFromEgress da classe TEAgent, responsável pela verificação do tipo de componente.

\section{Classe MPLSForwardingTable}

\section{1)Método reRouteToPrimaryPathLSP_OXC}

a) Responsável por identificar os lambdas, que correspondem à distância entre valores repetidos num padrão de onda, do LSP de backup (tanto de entrada quanto de saída), além de também identificar os lambdas dos LSPs primários que devem ser ativados, tanto de entrada e saída. Uma vez feito isto, desconecta o par de lambdas (entrada e saída) do LSP de backup e conecta o par de lambdas (entrada e saída) correspondente ao LSP primário. Este procedimento é realizado para LSPs ingressos.

b) No caso de LSPs egressos, certifica se o protocolo de sinalização sendo utilizado é o RSVP-TE. Se for invoca o método sendPrimaryPathOnToEgress (novo método criado) da classe $R S V P$.

Essas novas características implementadas nas classes acima citadas visam uma melhoria no simulador no que diz respeito às características de Engenharia de Tráfego já disponibilizadas com o acoplamento de um novo módulo de re-roteamento dinâmico de túneis LSPs.

\section{ANÁLISE DA IMPLEMENTAÇÃO}

\section{A. Cenário}

Nesta seção será apresentado um cenário de simulação para avaliar o módulo de re-roteamento dinâmico de LSPs no simulador. A topologia do cenário, ilustrada pela Figura 2, foi elaborada para que se tenham caminhos redundantes em que se possa utilizar o módulo tolerante a falhas de re-roteamento dinâmico de LSPs.



Fig. 2. Topologia utilizada na simulação 
Nessa topologia, tem-se uma rede com 17 nós e cada enlace óptico têm capacidade de 10GB. Os nós 5, 6, 7 e 8 atuam como LERs (Label Edge Routers-Roteadores de Rótulos de Borda) e os nós 9, 10,11, 12, 13, 14, 15 e 16 são OXCs, responsáveis por realizar a comutação óptica e comutação por rótulos. $\mathrm{O}$ nós $1,2,3$ e 4 são hosts conectados à rede e executam aplicações. No simulador, quem cria os caminhos comutados por rótulos é um no host que pode ou não estar conectado à topologia da rede simulada. Nesta simulação o nó que cria os túneis LSPs é o host 0.

O host 1 solicita tráfego do tipo UDP (User Datagram Protocol) para o host 3. Sendo assim, nesta simulação definiuse que o tráfego teria como origem o nó host 3 e o destino será o nó host 1 . O túnel LSP Primário é criado entre os LERs 6 e 8, porém para criar esse túnel deve-se primeiramente criar um LSP entre os nós OXCs 15, 9, 10 e 16. O túnel LSP Reserva para o túnel LSP Primário é criado entre os LERs 6 e 8, porém para criar esse túnel de reserva deve-se primeiramente criar um LSP entre os nós OXCs 15, 13, 14 e 16.

\section{B. Resultados da Simulação}

Partiu-se para a realização de simulações com o objetivo de avaliar o módulo de re-roteamento dinâmico, comparando resultados de eventos de falhas e recuperação com o novo módulo inserido no simulador e com a distribuição padrão GLASS, sem o novo módulo. A simulação foi repetida cinco vezes com tráfego aleatório e não foram usados geradores diferentes para cada variável aleatória da simulação.

Simulou-se um evento de falha no LSP primário entre os nós 9 e 10 e a falha ocorre no instante 40s da simulação e o enlace retorna à condição inicial no instante 50s. Um evento de falha também e simulado no LSP de reserva entre os nós 13 e 14 e ocorre no instante 60 s da simulação.

As Figuras 3 e 4 mostram o comportamento do parâmetro Throughput (vazão), que é a métrica que corresponde a quantidade de bytes recebidos pelo destino do túnel LSP entre os hosts 6 e 8 para os eventos descritos. Na primeira é apresentado o comportamento do Throughput do Túnel LSP entre os host 6 e 8 sem o novo módulo de re-roteamento inserido no simulador.

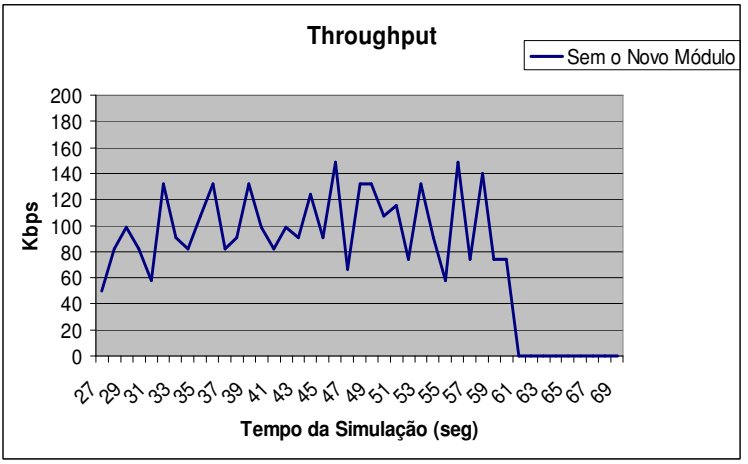

Fig. 3. Throughput do Túnel LSP Primário sem o Novo Módulo

No instante 40s decorrido da simulação ocorre um evento de falha no segmento entre os OXCs 9 e 10 do Túnel LSP Primário. Nesse momento o tráfego do túnel LSP Primário é roteado para seu túnel LSP de Reserva fazendo com que um dos requisitos de engenharia de tráfego seja estabelecido.

Decorrido 10 s da existência do evento de falha no segmento do túnel LSP Primário, esse é restabelecido no instante 50s. Como o cenário é simulado sem o novo módulo após o restabelecimento do túnel LSP Primário o tráfego não é reroteado para o Túnel LSP Primário.

Decorrido $60 \mathrm{~s}$ foi simulado um evento de falha no segmento entre os nós OXCs 13 e 14 do túnel LSP de Reserva. Como o tráfego não foi re-roteado para o túnel LSP Primário quando do seu restabelecimento, a Figura 3 mostra que existe uma diminuição acentuada no parâmetro Throughput do tráfego entre os host 6 e 8.

Na Figura 4 é apresentado o comportamento do Throughput do Túnel LSP entre os host 6 e 8 com o novo módulo de reroteamento inserido no simulador. A Figura 4 mostra que após o restabelecimento do segmento entre os OXCs 9 e 10 no instante 50s decorridos da simulação, o tráfego é re-roteado para o LSP Primário fazendo com que o segundo requisito de engenharia de tráfego seja estabelecido.



Fig. 4. Throughput do Túnel LSP Primário com o Novo Módulo

Como no instante 60s é estabelecido um evento de falha no segmento entre os OXCs 13 e 14 do túnel LSP de Reserva, pode ser observado que o comportamento do valor de Throughput entre os host 6 e 8 ficou dentro dos parâmetros aceitáveis.

Nas Figuras 5 e 6 é apresentada a quantidade de pacotes ópticos no Túnel LSP Primário entre os host 6 e 8 após as ocorrências dos eventos sem e com o novo módulo de reroteamento inserido no simulador.



Fig. 5. Quantidade de pacotes ópticos sem o Novo Módulo

$\mathrm{Na}$ Figura 5 podemos observar uma redução total na 
quantidade de pacotes após o primeiro evento de falha que ocorre no instante $40 \mathrm{~s}$.

Na Figura 6 também é apresentada a quantidade de pacotes ópticos no Túnel LSP entre os host 6 e 8 após as ocorrências dos eventos, porém com o novo módulo de re-roteamento inserido no simulador.

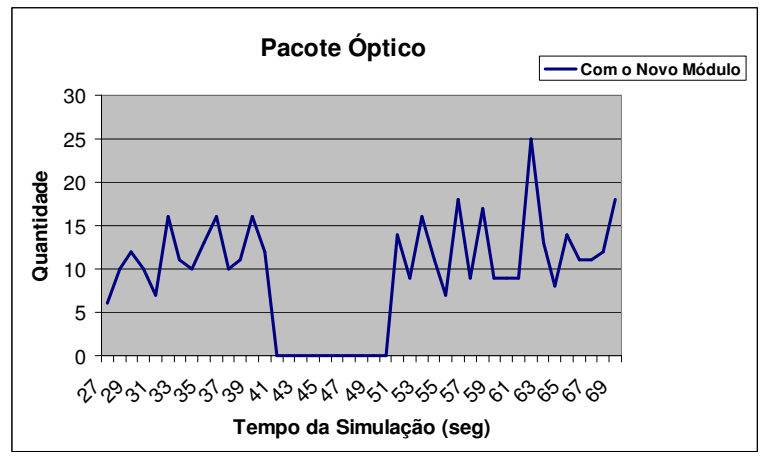

Fig. 6. Quantidade de pacotes ópticos com o Novo Módulo

Com o novo módulo de re-roteamento dinâmico de LSPs acoplado no simulador, a quantidade de pacotes perdidos após as ocorrências diminuir sensivelmente ficando dentro dos parâmetros aceitáveis.

A análise básica dos resultados obtidos nas duas simulações procura evidenciar o fato de que o re-roteamento dinâmico de LSPs após um evento de recuperação, obtida através da introdução do novo módulo basedo nos objetos do protocolo RSVP-TE, permite um melhor balanceamento do uso dos recursos da rede, no caso caracterizado pela uso racional dos túneis LSPs.

\section{CONSIDERAÇÕES E TRABALHOS FUTUROS}

O objetivo deste artigo foi descrever um novo módulo implementado no simulador GLASS para redes Ópticas MPLS/GMPLS. Este novo módulo tem como característica principal, executar o re-roteamento dinâmico de LSPs primários em caso do seu restabelecimento. $\mathrm{Na}$ última distribuição do GLASS, Versão 2.0.2, que é a utilizada neste trabalho, não existe a função de re-roteamento de túneis LSPs em cenários de redes MPLS/GMPLS. Dificultando assim, a implementação de requisitos de Engenharia de Tráfego como parâmetro de QoS (Quality of Service), tais como: throughput, delay, jitter, taxa de perda de pacotes e taxa de erro.

Assim o novo módulo visa agregar ao simulador a função de re-roteamento dinâmico de LSPs primários utilizando características das mensagens de sinalização do protocolo RSVP-TE. Portanto, este artigo, através do acoplamento da nova funcionalidade, fornece uma outra alternativa de ferramenta de simulação para o estudo fim-a-fim do nível de qualidade de serviço em redes ópticas com as tecnologias MPLS/GMPLS.

No decorrer deste trabalho, verificou-se a necessidade de avaliar o novo módulo com cenários mais complexos a fim de provar o correto funcionamento da implementação realizada, bem como aprimorar algumas outras funcionalidades no simulador como a utilização de gerenciamento baseado em políticas para provisionamento dinâmico de DiffServ e GMPLS. O aprimoramento do módulo de plotagem dos gráficos em cima dos resultados gerados pela simulação e a adição de técnicas de simulação estocástica também se fazem necessários.

\section{REFERÊNCIAS}

[1] Jain, R. "The Art of Computer SystemsPerformance Analysis: Techiques for Experimental Design, Mesasurement, Simulation and Modeling", John Wisley \& Sons, New York, 1991, 685p.

[2] OPNET, "OPNET Web Site". Disponível em: http://www.opnet.com. Acessado em Março de 2008

[3] VINT Project, The Virtual InterNetwork TestBed. A Collaboration among USC/ISI, Xerox PARC, LBNL, and UCB. Url: http://www.isi.edu/nsnam/vint/index.html. Acessado em Março de 2008.

[4] Rosen, E.; Viswanathan, A.; Callon, R. Multiprotocol Label Switching Architecture. RFC 3031. Janeiro, 2001

[5] Mannie, E. (Editor). Generalized Multi-Protocol Label Switching (GMPLS) Architecture. RFC 3945. Outubro, 2004.

[6] GLASS Overview - Version: Draft 1.0. Disponível em: http://wwwx.antd.nist.gov/glass/doc/html/overview/GLASS_Overview.htm Acessado em: Março de 2008.

[7] NIST, "National Institute of Standards and Technology Web Site". Disponível em: http://www.nist.gov/. Acessado em Março de 2008.

[8] MERLin, "Modeling Evaluation and Research of Lightwave Network Web Site". Disponível em: http://w3.antd.nist.gov/Hsntg/prd_merlin.html. Acessado em Março de 2008.

[9] SSFNet, "Scalable Simulation Framework Network Web Site" Disponível em: http://www.ssfnet.org/homePage.html. Acessado em Março de 2008

[10] DML, "Domain Modeling Language - Reference Manual". Disponível em http://www.ssfnet.org/SSFdocs/dmlReference.html. Acessado em Março de 2008

[11] Ingham, D. B.; Parrington, G. D. "A Wide-Area Network Emulation Tool” Computing System, v.7, n.3, pp. 313-332, 1994

[12] Rizzo, L. "Dummynet: A Simple Approach to the Evaluation of Network Protocols", ACM Computer Communication Review, v. 27, n.1, Jan. 1997.

[13] Leite, O. F. "ComFIRM - Injeção de Falhas de Comunicação Através da Alteração de Recursos do Sistema Operacional", Dissertação de Mestrado, CPGCC - UFRGS, Porto Alegre, Dezembro, 2000.

[14] Brakmo, S. L.; Peterson, L. L. "Experiences with Network Simulation", In ACM SIGMETRICS, Philadelphia, pp. 80-90, Maio, 1996.

[15] Little, C. M. "JavaSim Users Guide", Public Realease, Version 0.3. Disponível em http://javasim.ncl.ac.uk/. Acessado em Março de 2008.

[16] Fidalgo, J.; Kamienski, C.; Farias, T.; Dias, K.; Almeida, F.; Sadok, D.; Sadok, J. QNet - Um Simulador Gráfico de Tráfego IP para Redes Convergentes. $22^{\circ}$ Simpósio Brasileiro de Redes de Computadores SBRC 2004, maio 2004, Rio Grande do Sul.

[17] Ahn, G.; Chun, W. Architecture of MPLS Network Simulator (MNS). Technical Report, Departamento f Computer Engineering, Chungman University of Korea. 2002.

[18] Muhammad, H. H.; Barcellos, M. P. Simmcast: Uma Ferramenta de Simulação para Avaliação de Protocolos Multicast. REIC. Revista Eletrônica de Iniciação Científica, v. 2, n.2, p. 1-8, 2001.

[19] Nascimento, A.; Franklin, M.; Oliveira, M. Desenvolvendo Agentes Inteligentes para a Gerência Pró-Ativa de Redes ATM. $17^{\circ}$ Simpósio Brasileiro de Redes de Computadores - SBRC 1999, maio 1999, Bahia.

[20] Gonçalves, C.; Oliveira, A.; Andrade, R.; Castro, M.. Utilizando Redes Neurais para Predição de Falhas em Links de Redes Ópticas. Boletim Bimestral sobre Tecnologia de Redes, Rio de Janeiro, v. 7, 2003.

[21] Cunha, L. S.; Rochol, J.; Dalto, L.; Fernandes, R. H.; Mori, O. Plataformas de Simulação para Redes Fixas e Móveis: Características, Suporte, Instalação e Validação. In: 2nd International Information and Telecommunication Technologies Symposium, 2003, Florianópolis Proceedings of the 2nd International Information and Telecommunication Technologies Symposium, 2003. 